\title{
Factores asociados a etapas de cambio de comportamiento en fumadores cubanos
}

\author{
Patricia Varona Pérez, ${ }^{1}$ Mariela Rodríguez, ${ }^{1}$ Karen Alfonso, ${ }^{1}$ Mariano Bonet, ${ }^{1}$ \\ René Guillermo García, ${ }^{1}$ Niurys Fernández Larrea ${ }^{1}$ y Rosa Marina García Pérez ${ }^{1}$
}

RESUMEN Objetivo. Describir la distribución de los fumadores cubanos en las etapas iniciales del proceso de cambio — precontemplación y contemplación-, las características de estos fumadores y la relación entre dichas características y algunas variables de interés.

Métodos. Como parte de la Primera Encuesta Nacional de Factores de Riesgo y Actividades Preventivas de las Enfermedades Crónicas no Trasmisibles, realizada en 1996 en Cuba, se seleccionó una muestra probabilística por conglomerados en tres etapas con estratificación de las unidades primarias. Las unidades de la primera etapa fueron los distritos censales, seleccionados con probabilidades en proporción con el número de viviendas. Como estratos se consideraron las 14 provincias y los 169 municipios en que está dividido el país de acuerdo con la división político-administrativa vigente. En total se seleccionaron 4835 fumadores de 15 años de edad o más, a quienes se aplicó un cuestionario con variables sociodemográficas (edad, sexo, color de la piel, escolaridad y tipo de labor desempeñada con carácter permanente) y conductuales relacionadas con el tabaquismo (estado de fumador, conocimientos del efecto perjudicial del tabaco para la salud, edad a la que se comenzó a fumar, número de cigarrillos fumados diariamente, disposición a abandonar ese hábito y número de intentos de abandono), lo cual permitió ubicarlos en las etapas iniciales de un cambio conductual en relación con el tabaquismo. La probabilidad de estar en la etapa de precontemplación se modeló mediante regresión logística utilizando el método de las significaciones sucesivas. Los coeficientes estandarizados que aportó este modelo fueron utilizados para calcular el peso de las variables explicativas incluidas. Para el cálculo de las estimaciones y sus errores por muestreo se utilizaron los paquetes estadísticos SAS 6.12 y SUDAAN 7.5, teniendo en cuenta el diseño complejo de la muestra. Se estableció un nivel de significación de 0,05 .

Resultados. Según los resultados, $46,4 \%$ de los fumadores encuestados estaban en la etapa de precontemplación o falta de motivación para el cambio; las mujeres se mostraron más dispuestas que los hombres a abandonar la adicción; mientras mayor era el nivel de instrucción, el deseo de abandonarla era mayor; las personas vinculadas al trabajo estuvieron más motivadas para el cambio. A medida que aumentaron la edad de inicio de fumar, la cantidad de cigarrillos consumidos diariamente y los intentos de dejar de fumar, disminuyó la probabilidad de estar en la etapa de precontemplación.

Conclusiones. La proporción de fumadores en etapa de contemplación ubica a Cuba en una situación favorable para el desarrollo de intervenciones preventivas antitabáquicas eficaces. La mayoría de los factores identificados (edad de inicio de la adicción, cantidad de cigarrillos consumidos a diario e intentos de abandonar el hábito) pueden modificarse mediante acciones preventivas. La identificación de las etapas en el proceso de cambio y de los factores a ellas vinculados debe constituir una condición necesaria para el diseño y la aplicación de programas preventivos y de control del tabaquismo que aspiren a ser realmente eficaces.

Palabras clave Cese del tabaquismo, Cuba.

1 Instituto Nacional de Higiene, Epidemiología y Microbiología (INHEM), Ciudad de La Habana, Cuba. La correspondencia debe enviarse a: Patricia
Varona, Instituto Nacional de Higiene, Epidemiología y Microbiología, Infanta No. 1158 e/Clavel y Llinás, Centro Habana, Ciudad de La Habana,
Cuba. Teléfono: (537) 878 8479. Fax: (537) 662404. Correo electrónico: patriciav@inhem.sld.cu. 
La influencia del tabaquismo en la aparición de las enfermedades crónicas no trasmisibles ha sido reconocida en la literatura científica (1-3). Estas enfermedades ocupan los primeros lugares en el cuadro de morbilidad y mortalidad de Cuba (4) y constituyen un problema real de salud que afecta a $32 \%$ de la población cubana de manera activa y al resto de forma pasiva. Por lo tanto, desestimular la adicción al tabaco es un reto que es preciso asumir.

Desde la década de 1970, en el campo de la psicología de la salud se comenzó a desarrollar modelos para estimular el cambio de comportamiento. Según el modelo transteórico - que constituye una guía para el diagnóstico y la delimitación inicial del problema o actitud que se desea cambiar (5)—, esto se concibe como un proceso y se considera que las personas tienen diversas motivaciones o diferentes grados de disposición para pasar de una etapa a otra. En consecuencia, en los diferentes puntos del proceso pueden lograrse los beneficios de intervenciones ajustadas a cada etapa.

Las etapas conocidas por precontemplación, contemplación, preparación, acción y mantenimiento (5-7) describen los diversos momentos a lo largo de este proceso: cuando la persona no está motivada para el cambio, cuando lo planifica, lo ejecuta y lo mantiene. Esto que parece ser un proceso continuo no siempre se manifiesta de forma secuencial, de modo que pueden ocurrir retrocesos o saltos de etapas.

Conocer este diagnóstico puede resultar muy útil, pues las personas que se encuentran en la etapa de contemplación cuentan con la motivación inicial para el cambio. De igual manera, es importante identificar la relación de estas etapas con factores que puedan obstaculizar o facilitar el cambio, ya que pueden influir en su desarrollo y además permiten predecir la deshabituación tabáquica. Por esto, antes de ejecutar acciones de educación o prevención contra el tabaquismo se debe definir la etapa de cambio del comportamiento en que se encuentran los fumadores, pudiéndose así hacer los ajustes necesarios en función de las motivaciones y la condición psicosocial de la población a la que van dirigidas esas acciones. Esto permitirá diseñar estrategias más eficaces para modificar la conducta y lograr que el cambio se mantenga.

No se conocen otros estudios realizados en Cuba que utilicen este tipo de información en programas y campañas preventivas dirigidos a estimular el paso de una etapa a otra, de manera que el fumador reciba ayuda mientras abandona ese hábito y se potencie la efectividad de las acciones. El objetivo de este trabajo es describir la distribución de los fumadores cubanos en las etapas iniciales del proceso de cambio - precontemplación y contemplación-, las características de estos fumadores y la relación entre dichas características y algunas variables de interés.

\section{MATERIALES Y MÉTODOS}

En 1996 se realizó en Cuba la Primera Encuesta Nacional de Factores de Riesgo y Actividades Preventivas de las Enfermedades Crónicas no Trasmisibles. El objetivo de ese estudio transversal representativo de la población urbana de 15 años de edad o más fue evaluar los principales factores de riesgo asociados con enfermedades crónicas no trasmisibles y sus características epidemiológicas. En esa encuesta se incluyó una serie de preguntas relacionadas con el tabaquismo: si era fumador, conocimientos sobre el efecto perjudicial del tabaco sobre la salud, edad a la que comenzó a fumar, número de cigarrillos fumados diariamente, disposición a abandonar el hábito y número de intentos anteriores por abandonarlo. También se incluyó un bloque de preguntas sobre variables sociodemográficas: edad, sexo, color de la piel, escolaridad y tipo de labor desempeñada con carácter permanente.

Se seleccionó una muestra probabilística por muestreo por conglomerados en tres etapas con estratificación de las unidades primarias. Las unidades de la primera etapa fueron los distritos censales, seleccionados con probabilidades en proporción con el número de viviendas. Como estratos se consideraron las 14 provincias y los
169 municipios en que está dividido el país de acuerdo con la división político-administrativa vigente.

Dentro de cada municipio se seleccionó una muestra autoponderada. Para la estimación se realizó una estratificación por sexo y grupo de edad (15-19, 20-29, 30-39, 40-49, 50-59, 6064 y mayores de 64 años). La muestra fue de 4835 fumadores, lo que de acuerdo al diseño muestral equivaldría a 2263586 fumadores en la población cubana.

Dados el objetivo de la encuesta y la estructura de las preguntas, se decidió trabajar con las dos primeras etapas del proceso de cambio de comportamiento descrito por Prochaska y Di Clemente (5-6).

Las definiciones operacionales de las variables empleadas son las siguientes:

- Fumadores en etapa de precontemplación (definida con valor 1): fumadores que declararon no tener deseos de abandonar la adicción. Respondieron negativamente a la pregunta "¿desea usted dejar de fumar?"

- Fumadores en etapa de contemplación (definida con valor 0 ): fumadores que declararon estar dispuestos a dejar de fumar. Respondieron afirmativamente a la pregunta anterior.

- Fumador: toda persona que declaró haber consumido al menos un derivado del tabaco al día durante un período mínimo de un mes y que fumaba en el momento de la encuesta. Se tuvieron en cuenta los fumadores diarios debido a su elevada frecuencia en la población cubana.

- Nivel de escolaridad: primaria (hasta primaria terminada), secundaria (obrero calificado o secundaria básica terminada), media superior (preuniversitario o estudio técnico medio terminado), o graduados universitarios.

- Situación laboral: trabajador, en busca de trabajo, pensionado, ama de casa o estudiante.

- Conocimiento: esta variable se construyó a partir de las respuestas a la pregunta sobre la relación entre el tabaquismo y la salud, asignando valores entre 1 y 6 , según las respuestas. 
Se evaluó la asociación entre las etapas del proceso de cambio de comportamiento estudiadas y las variables de sexo, situación laboral, edad promedio, consumo diario de cigarrillos, intentos de abandono de la adicción y nivel de conocimientos, para lo que se construyeron tablas de contingencia y se aplicó la prueba de ji al cuadrado.

La probabilidad de estar en la etapa de precontemplación se modeló mediante regresión logística, utilizando el método de las significaciones sucesivas. Los coeficientes estandarizados que aportó este modelo fueron utilizados para calcular el peso de las variables explicativas incluidas. Para el cálculo de las estimaciones y sus errores por muestreo se utilizaron los paquetes estadísticos SAS 6.12 y SUDAAN 7.5 , teniendo en cuenta el diseño complejo de la muestra. Se estableció un nivel de significación de 0,05.

\section{RESULTADOS}

Entre los fumadores predominaron los hombres (61,2\%). En general, la edad promedio en que se inició el hábito de fumar fue de 17 años. Aunque los inten-

CUADRO 1. Características de la muestra de fumadores cubanos $(n=4835)$

\begin{tabular}{lc}
\hline \multicolumn{1}{c}{ Variable } & Valor \\
\hline Edad promedio (años) & 44 \\
Proporción de hombres (\%) & 63,2 \\
Fumadores en etapas de cambio (\%) & \\
Contemplación & 53,64 (IC95\%: 51,64 a 55,64) ${ }^{a}$ \\
Precontemplación & 46,36 (IC95\%: 44,36 a 48,36) \\
Consumo promedio diario de cigarrillos (unidades) & 13 \\
Edad promedio de inicio de la adicción (años) & 17 \\
Intentos de abandonar la adicción (media) & 1,6 \\
\hline
\end{tabular}

a IC95\%: intervalo de confianza de 95\%.

tos de abandonar la adicción fueron 1,6 en promedio, la proporción de fumadores que consideraban la posibilidad de abandonar el tabaquismo (fumadores en la etapa de contemplación) fue mayor que la de los que no deseaban el cambio (cuadro 1). Los intentos de abandono fueron más numerosos en el grupo que se encontraba en la etapa de contemplación (cuadro 2).

Las mujeres se mostraron más dispuestas a abandonar el tabaquismo que los hombres (cuadro 2), aunque no se encontraron diferencias estadísticamente significativas. El promedio de edad fue ligeramente superior en el grupo que se encontraba en la etapa de precontemplación. En relación con el nivel de escolaridad, se encontró que los fumadores con estudios secundarios, medios superiores y universitarios estaban más dispuestos a dejar de fumar que los que tenían solamente estudios del nivel primario. Entre los que deseaban dejar de fumar hubo una mayor proporción de trabajadores y estudiantes, así como de amas de casa; mientras que los pensionados fueron los que menos deseos de cambio declararon.

En el cuadro 3 se presentan, en orden descendente según su influencia, las variables asociadas a la probabilidad de estar en la etapa de precontemplación. Por cada año que aumentó la edad se

CUADRO 2. Comportamiento de las variables de interés en las etapas iniciales de cambio, Cuba

\begin{tabular}{|c|c|c|c|c|}
\hline Variable & $\begin{array}{c}\text { Etapa de } \\
\text { contemplación }\end{array}$ & $\operatorname{IC} 95 \% \mathrm{a}$ & $\begin{array}{c}\text { Etapa de } \\
\text { precontemplación }\end{array}$ & IC95\% \\
\hline \multicolumn{5}{|l|}{ Sexo (\%) } \\
\hline Mujeres & 56,99 & 53,72 a 60,27 & 43,00 & 39,72 a 46,27 \\
\hline Hombres & 51,68 & 49,11 a 54,26 & 48,31 & 45,74 a 50,89 \\
\hline \multicolumn{5}{|l|}{ Nivel de escolaridad (\%) } \\
\hline Primario & 47,87 & 44,60 a 51,11 & 52,12 & 48,84 a 55,40 \\
\hline Secundario & 54,48 & 51,00 a 58,17 & 45,41 & 41,83 a 49,00 \\
\hline Medio superior & 57,63 & 53,45 a 61,80 & 42,37 & 38,19 a 46,54 \\
\hline Superior & 57,36 & 50,32 a 64,41 & 42,63 & 35,59 a 49,67 \\
\hline \multicolumn{5}{|l|}{ Situación laboral (\%) } \\
\hline Trabajador & 55,96 & 53,39 a 58,53 & 44,03 & 41,46 a 46,61 \\
\hline En busca de trabajo & 48,02 & 34,83 a 61,21 & 51,97 & 38,78 a 65,16 \\
\hline Pensionado & 43,44 & 38,54 a 48,34 & 56,55 & 51,65 a 61,45 \\
\hline Ama de casa & 54,50 & 49,38 a 59,61 & 45,50 & 40,38 a 50,61 \\
\hline Estudiante & 64,25 & 47,02 a 81,47 & 35,75 & 18,52 a 52,97 \\
\hline Otra & 50,85 & 41,31 a 60,39 & 49,14 & 39,60 a 58,69 \\
\hline Edad promedio (años) & 41,80 & 41,03 a 42,56 & 44,66 & 43,66 a 45,66 \\
\hline Consumo promedio diario de cigarrillos (unidades) & 13,22 & 12,74 a 13,69 & 12,32 & 11,77 a 12,88 \\
\hline Intentos de abandono (media) & 2,34 & 2,24 a 2,47 & 0,71 & 0,62 a 0,80 \\
\hline \multicolumn{5}{|l|}{ Nivel de conocimiento de la relación entre } \\
\hline el tabaquismo y la salud (media) ${ }^{b}$ & 2,66 & 2,59 a 2,73 & 2,57 & 2,46 a 2,59 \\
\hline
\end{tabular}

a IC95\%: intervalo de confianza de $95 \%$.

${ }^{\mathrm{b}}$ Evaluado en una escala de 1 a 6 , donde 6 representa un mayor conocimiento. 
CUADRO 3. Grado de asociación de diversas variables con la etapa de precontemplación según los resultados del modelo de regresión logística con ajustes, Cuba

\begin{tabular}{lcccc}
\hline \multicolumn{1}{c}{ Variables } & Coeficiente & $\begin{array}{c}\text { Coeficientes } \\
\text { estandarizado }\end{array}$ & $\begin{array}{c}\text { Razón de } \\
\text { posibilidades }\end{array}$ & IC95\%a \\
\hline $\begin{array}{l}\text { Intercepto } \\
\text { Edad (años) }\end{array}$ & 0,52 & - & - & - \\
$\begin{array}{l}\text { Edad a la que comenzó a fumar } \\
\quad \text { (años) }\end{array}$ & 0,01 & 3,40 & 1,01 & $1,01-1,02$ \\
$\begin{array}{l}\text { Consumo diario de cigarrillos } \\
\quad \text { (unidades) }\end{array}$ & $-0,02$ & $-2,89$ & 0,98 & $0,97-0,99$ \\
$\begin{array}{l}\text { Intentos de abandonar el hábito (No.) } \\
\text { (Non }\end{array}$ & $-0,01$ & $-2,04$ & 0,99 & $0,98-1,00$ \\
\hline
\end{tabular}

a IC95\%: intervalo de confianza de $95 \%$.

incrementó en $1 \%$ la probabilidad de estar en la etapa de precontemplación. Como se puede observar, a medida que aumentaron la edad en que se empezó a fumar, la cantidad de cigarrillos consumidos a diario y los intentos de dejar de fumar, disminuyó la probabilidad de estar en la etapa de precontemplación. Por cada año que aumentó la edad en que empezó la adicción se redujo en $2 \%$ la probabilidad de no desear el cambio. Por cada cigarrillo consumido diariamente disminuyó en 1\% la probabilidad de no desear el cambio, la cual se redujo en $58 \%$ por cada intento de abandono. No hubo diferencias significativas en la distribución de los encuestados en la etapa de precontemplación según el color de la piel.

La distribución de los fumadores en las provincias cubanas según las etapas de cambio de comportamiento y su relación con la prevalencia de tabaquismo en esos territorios mostró que en las regiones central y oriental del país la proporción de fumadores en precontemplación fue menor que en la parte occidental. Seis provincias (Pinar del Río, La Habana, Ciudad de La Habana, Matanzas, Camagüey y Guantánamo) y el municipio especial de Isla de la Juventud excedieron la proporción nacional de fumadores en la etapa de precontemplación. La Habana, en la región occidental, fue la provincia con mayor proporción de fumadores en la etapa de precontemplación, mientras que Holguín, en la región oriental, presentó la situación más favorable (figura 1).

No se encontró ninguna correlación entre la prevalencia de tabaquismo y el porcentaje de fumadores en la etapa de precontemplación, por provincias (coeficiente $r$ de Pearson $=-0,12 ; P=0,6694$ ). En las diferentes regiones del país se encontraron resultados similares.

\section{DISCUSIÓN}

En comparación con otros países (89), Cuba cuenta con una situación más favorable para el desarrollo de intervenciones antitabáquicas por la elevada proporción de fumadores en la etapa de contemplación. En un estudio realizado en la población de Ginebra, Suiza, la proporción de fumadores en la etapa de precontemplación excedió en $28 \%$ la encontrada en esta investigación, en tanto que la reportada en los Estados Unidos de América fue menor en $6 \%$.

La mayor proporción de mujeres dispuestas al cambio confirma lo encontrado por otros autores que hallaron entre las mujeres una mayor percepción de riesgo (10-11). Etter et al. (8), sin embargo, encontraron una mayor proporción de mujeres en la etapa de precontemplación. Esta discrepancia pudiera estar relacionada con el elevado nivel educacional de la mujer cubana y sugiere que, además de las etapas de cambio, es importante tener en cuenta el sexo y la percepción particular de cada uno de los grupos a los que se dirigen las acciones. Toda esta información ayuda a orientar de manera diferenciada las acciones preventivas que se deben desarrollar para lograr el cambio de comportamiento.

En relación con el nivel de instrucción, los resultados obtenidos coinciden con los de investigaciones anteriores (12-14), en las que se señala que la escolaridad es una de las variables que influyen en la receptividad a los mensajes y en la disposición para emprender acciones preventivas. Esto se explica por el hecho de que la capacidad de una persona para procesar información varía según su nivel de instrucción. El porcentaje de personas en la etapa de

FIGURA 1. Distribución de fumadores en etapa de precontemplación y prevalencia de fumadores, por provincias, Cuba

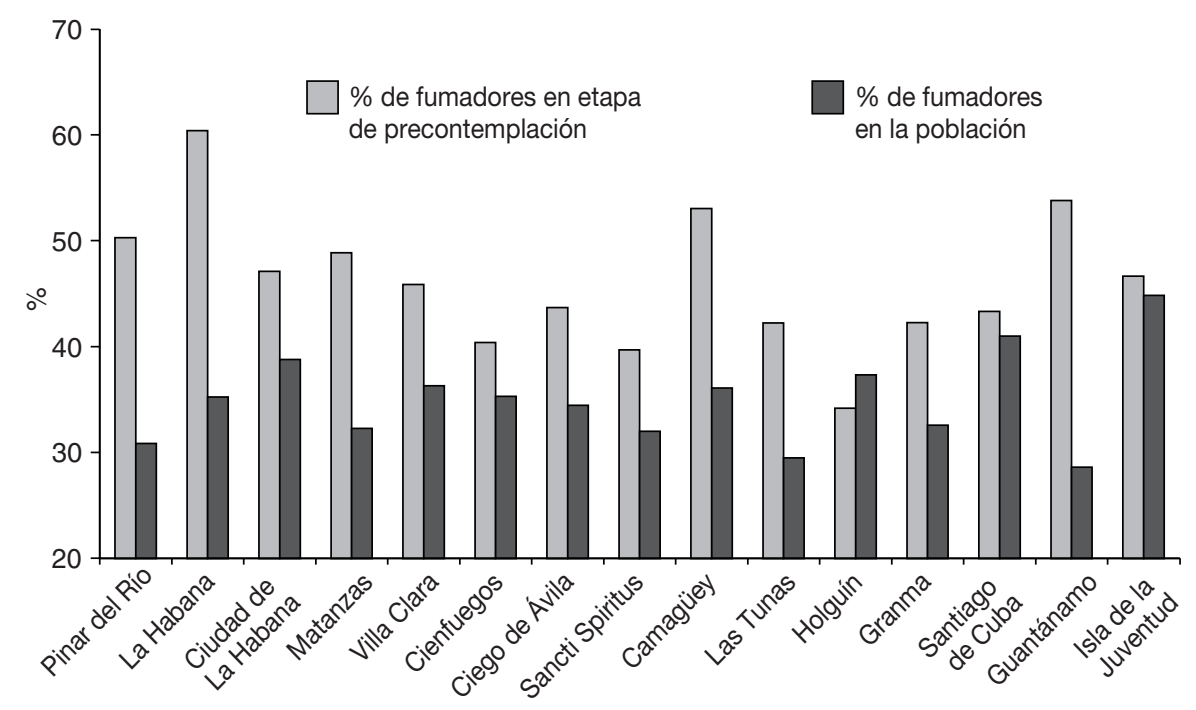


contemplación encontrado en el presente estudio pudiera estar relacionado con los temas y mensajes incluidos en el currículo escolar desde la enseñanza primaria, con un refuerzo progresivo en niveles superiores y a través de los medios de comunicación masivos.

El malestar y el ocio de las personas que no tienen vínculos laborales pudieran estimular el hábito del tabaquismo (13). Sin embargo, esta hipótesis no fue objetivo de este estudio y debe ser explorada en otras investigaciones. En relación con las personas de edad avanzada, su resistencia característica al cambio y su deseo de mantener sus hábitos pudieran explicar su menor disposición a abandonar el tabaquismo.

El inicio precoz del consumo de cigarrillos es un fuerte factor pronóstico en relación con la dependencia de la nicotina (14), y la probabilidad de abandonar el consumo regular aumenta en la medida en que aumenta la edad en que ocurren los procesos de experimentación y consolidación del consumo regular del tabaco (15).

Aunque el nivel de adicción, tanto psicológica como física, es mayor cuando el consumo de cigarrillos aumenta, la relación inversa encontrada en nuestro estudio entre el consumo de cigarrillos y la probabilidad de estar en la etapa de precontemplación pudiera explicarse porque un mayor consumo puede tener para la persona otras consecuencias negativas, como los daños a su salud y su economía individual y familiar, tal como se ha señalado en diversas publicaciones (16-17).

El número de intentos de abandonar la adicción al tabaco está directamente relacionado con la etapa de precontemplación y confirma los resultados de otras investigaciones (14). Los intentos de abandonar el tabaquismo pueden considerarse un factor pronóstico de la intención de cambio, con independencia de su fracaso. Sin embargo, hay que tener en cuenta que la percepción de una persona se basa principalmente en la experiencia vivida (18-20) y esto pudiera, en algunos casos, reducir las posibilidades de volver a concebir la idea de cambio, al pensar que no podrán lograrlo, que no son capaces de cambiar.

Por eso es muy importante en los inicios de las campañas anticipar la probabilidad de fracaso, sin que ello signifique que sea imposible concluir el proceso. Si se admite la posibilidad de fracasar en el intento, cada fracaso llevará a reflexionar sobre las causas del mismo con vistas a mejorar en el próximo intento y a aumentar la probabilidad de lograr los resultados deseados. De esta forma, cuanto más intentos se hagan, más posibilidades habrá de lograr el cambio (21).

Un aporte de este trabajo consiste en haber estudiado el grado de motivación para abandonar la adicción en las diferentes provincias de Cuba, lo que debe contribuir a la implementación y evaluación de acciones preventivas que respondan a las necesidades y realidades a escala local. Las diferencias observadas entre las provincias probablemente no se atribuyen a diferencias en la interpretación del cuestionario, ya que este fue aplicado en todo el país con igual metodología. La falta de asociación entre el porcentaje de fumadores en etapa de precontemplación y el porcentaje de fumadores por provincias contrasta con resultados obtenidos en otro estudio sobre la distribución de fumadores por etapas de cambio (8). En esa investigación se ob- servó una asociación entre esas variables, aunque no se logró identificar el sentido de la misma ni descartar la influencia de factores de confusión. La investigación de las diferencias entre regiones merece la mayor atención, ya que permitiría identificar las características locales, ya sean económicas, psicológicas o sociales, que pudieran explicar los resultados obtenidos en este estudio.

\section{CONCLUSIONES}

La proporción de fumadores que se encuentra en la etapa de contemplación ubica a Cuba en una situación favorable para el desarrollo de intervenciones preventivas antitabáquicas, hecho que se potencia por las características del sistema cubano de salud, que es único para todo el país y tiene amplia cobertura y total accesibilidad. Se deben identificar las etapas de cambio de comportamiento de los fumadores y diseñar programas de prevención y control que contribuyan a aumentar la motivación para el cambio y a crear conciencia de la necesidad de abandonar el tabaquismo.

La mayoría de las variables asociadas a la etapa de precontemplación (edad de inicio de la adicción, cantidad de cigarrillos consumidos a diario y número de intentos de abandonar el hábito de fumar) son susceptibles de ser modificadas mediante acciones preventivas eficaces, por lo que la identificación de las etapas del proceso de cambio y los factores vinculados a ellas deben constituir una condición necesaria para diseñar y adoptar programas preventivos y de control del tabaquismo que aspiren a ser realmente eficaces.

\section{REFERENCIAS}

1. Warren CW, Riley L, Asma S, Eriksen MP, Green L, Blanton C, et al. Tobacco use by youth: a surveillance report from the Global Youth Tobacco Survey Project. Bull WHO 2000;78:868-876.

2. Fiore M, Bailey W, Cohen S, Dorfman S, Goldstein M, Gritz E, et al. Treating tobacco use and dependence. Clinical Practice Guideline. Rockville, MD: U.S. Department of Health and Human Services; 2000.

3. National Cancer Institute. Health effects of exposure to environmental tobacco smoke: the report of the California Environmental Protection Agency. Bethesda, MD: U.S. Depart- ment of Health and Human Services, National Institutes of Health; 1999. (Smoking and Tobacco Control Monograph No. 10).

4. Cuba, Ministerio de Salud Pública. Anuario estadístico. La Habana: MINSAP; 2000.

5. Glanz K, Rimer BK, Sutton SM. Teorías de un vistazo: guía para la práctica de la promoción 
de la salud. Modelos y teorías de comunicación en salud. Promoción de la salud. División de Promoción y Protección de la Salud. Washington, D.C.: Organización Panamericana de la Salud; 1996. (Reproducción de Documentos Serie No. 19).

6. Soto M, Valderrama J, Balcázar $\mathrm{H}$. El proceso de abandono del tabaquismo: teoría, investigación y práctica. Gac Sanit 2001;15(Supl. 4): 49-54.

7. Pardell H, Saltó E, Salleras L. Manual de diagnóstico y tratamiento del tabaquismo. Madrid: Editorial Médica Panamericana; 1996.

8. Etter JF Perneger T, Ronchi A. Distributions of smokers by stage: international comparison and association with smoking prevalence. Prev Med 1997;26:580-585.

9. Etter JF, Perneger T. Associations between the stages of change and the pros and cons of smoking in a longitudinal study of Swiss smokers. Addict Behav 1999;24:419-424.

10. Flynn J, Slovic P, Mertz C. Gender, race and perception on environmental health risk. Risk Anal 1994;14(6):22-24.
11. Donald M, Fleming R. Risk perception reporting. Risk Anal 1996;16(6):58-62.

12. Costa M, López E. Salud comunitaria. Barcelona: Martínez Roca; 1986.

13. Kato I, Tominaga S, Suzuki T. Lifestyle differentials by occupational group. NipponKoshu-Eisei-Zasshi 1992;39(11):830-838.

14. Cabrera A. Etapas de cambio en consumidores de cigarrillo en Zarzal, Colombia, 1999. Revista de la Facultad Nacional de Salud Pública 2001;19:33-41.

15. Jackson C, Henriksen L, Dickinson D, Messer L, Roberston S. A longitudinal study predicting patterns of cigarette smoking. Health Educ Behav 1998;25:436-447.

16. World Health Organization. Guidelines for controlling-monitoring tobacco epidemic. Geneva: WHO; 1998.

17. Varona P, Soto G, García R, Bonet M, Álvarez $\mathrm{M}$, Cordero I. Costos de atención a la salud de fumadores y no fumadores en Ciudad de la Habana y Santiago de Cuba, 2000-2001. Memorias de la Convención Internacional Salud Pública 2002, Ciudad de La Habana 1-4 de mayo; 2002. (Publicación electrónica CD ISBN 959 7164-08-6).

18. Lovelle R. La psiquis en la determinación de la salud. La Habana: Científico-Técnica; 1989.

19. Orbell S, Sheeran P. Motivational and volitional processes in action initiation: a field study of the role of implementation intentions. J Appl Soc Psychol 2000;30:780-797.

20. Stajkovic A, Luthans, F. Self-efficacy and work-related performance: a meta-analysis. Psychol Bull 1998;124:240-261.

21. Sheeran P, Norman P. Evidence that intentions based on attitudes better predict behaviour than intentions based on subjective norms. Eur J Soc Psychol 1999;29:403-406.

Manuscrito recibido el 19 de septiembre de 2002. Aceptado para publicación, tras revisión, el 31 de marzo de 2003

ABSTRACT Objective. To describe the distribution of Cuban smokers in the first two stages of the process of behavior change (precontemplation (that is, no desire to quit smoking) and contemplation (thinking about quitting)), the characteristics of these smokers, and the relationship between these characteristics and some variables of interest.

Factors associated with stages of behavior change Methods. The First National Survey of Risk Factors and Chronic Non-communicable Disease Prevention Activities was carried out in Cuba in 1996. As a part of that Survey, a probability sample was selected using three-stage clustering, with stratification of the primary units, which were the census districts in the 14 provinces and $169 \mathrm{mu}-$ nicipalities that make up the country. The census districts were selected in proportion to their number of dwellings. A total of 4835 smokers 15 years old or older were selected to answer a questionnaire on sociodemographic variables and smoking-related behaviors. The sociodemographic variables included age, sex, skin color, level of schooling completed, and work status (working, looking for work, retired, housewife, or student). The smoking-related behaviors included knowledge of tobacco's harmful effects on health, age when smoking began, number of cigarettes smoked daily, willingness to give up the habit, and the number of attempts made to quit smoking. The questionnaire information made it possible to place the smokers in one or the other of the two initial stages of smoking behavior change. The probability of being in the precontemplation stage was modeled through logistic regression. The standardized coefficients that the model produced were used to calculate the weight of the explanatory variables included in the model. To calculate the estimates and their errors, the SAS 6.12 and SUDAAN 7.5 statistical software packages were used, taking into account the complex design of the sample. The level of significance was set at 0.05 .

Results. We found that $46.4 \%$ of the smokers surveyed were in the precontemplation stage, and $53.6 \%$ were in the contemplation stage. Women were more prepared to quit the habit than were men. The more formal the education that a person had, the stronger was the desire to quit smoking. Persons who were retired were the ones least likely to be considering giving up smoking. The probability of being in the precontemplation stage decreased with an increase in the age of beginning to smoke, the number of cigarettes smoked daily, and the number of attempts made to quit smoking. Conclusions. The proportion of Cuban smokers in the contemplation stage places the country in a favorable position for developing effective smoking prevention interventions. The majority of the factors identified (age when smoking began, number of cigarettes consumed daily, and number of attempts to give up the habit) can be modified through preventive actions. Identifying the stages in the process of change and the factors linked to them are necessary for designing and implementing effective smoking prevention and control programs. 